\title{
Analysis of Captured Data on a Typical Tcp Connection
}

\author{
A. S. Mindaudu ${ }^{1}$ and I. G. Saidu ${ }^{2}$ \\ ${ }^{I}$ Sokoto Energy Research Centre, Usmanu Danfodiyo University, Sokoto. \\ ${ }^{2}$ Dept. of Physics, Usmanu Danfodiyo University, Sokoto
}

\begin{abstract}
Various data capture tools are in existence today for either intrusive or non-intrusive capturing of data packets. The raw packet trace obtained with such capture tools can be used to determine a lot of information concerning a connection. Usually the data capture tools give insight into the behaviour of the traffic patterns, congestion window evolution and control. Through the possible analysis of the captured data, possible areas of network improvement could be established. One of such capturing tools, Wireshark, was used to capture some packets on an established TCP connection between a client with an IP address 192.168.0.140 and a distant server having IP address 128.227.74.66. In this paper an analysis of the captured data is presented. Phenomenon such as congestion window evolution in the connectionwasobserved on the time sequence graph which also gave an indication of the lost packets in the transmission.These lost packets were identified through the repeated ACKs on the relevant frames. With the captured data, the round trip time of $177 \mathrm{~ms}$ was calculated, and the average bottleneck bandwidth obtained was $91.97 \mathrm{Mbps}$ which compared favourably with the expected network bandwidth of $100 \mathrm{Mbps}$.In the captured data, a TCP-out-of-order packet was identified with a time stamp of 2.245038 indicating a lost packet.
\end{abstract}

Keywords :TCP, congestion window, bottleneck bandwidth, round trip time,

\section{Introduction}

Wireshark, Earthreal, TCP dump etc. are some of the few examples of data capture tools from which detailed analysis of a connection can be carried out. Using Wireshark several data packets were captured on a TCP connection between a client on IP address 192.168.0.140 in a network located at the University of Plymouth in the UKand a server at the University of Florida in the USA on IP address 128.227.74.66.

\section{TCP Connection Establishment and Closure}

In order to establish the TCP connection with the server and subsequently initiate data transfer, the client sends a TCP segment referred to as the SYN segment to the server. At this point in time no applicationlayer data is sent along with the packet number 1 , but the TCP segment sent also has a sequence number 0 which is chosen by the client. This is seen in packet number 1 in Trace 1 . So, by this time the server has the SYN and the Seq. number from the client. After receiving the TCP SYN segment from the client, the server then sends a packet to the client (i.e. packet number 2 in Trace 1). This time the server sends its initial sequence number which is 0 . The ACK flag is set to 1 implying that in the next packet coming from the client; it is expecting a sequence number 1 . At this point also, no application-layer data is sent.

The client having received the SYN ACK sends another TCP segment to the server. This time the server increments its sequence number to 1 and also sets ACK to 1 , thus signifying that the TCP connection has been established. Up to this time, however, there is still no application-layer data sent.

With these first three packets, the TCP connection between the server (IP 128.227.74.66) and the client (IP 192.168.0.140) is established. It is noted from the trace that both the client and the server set their receive windows to 65535 bytes.

The first three packets of the capture in Trace 1 describe the TCP connection set up. With the transmission of the first three packets, the client and server start exchanging data. The client first requests for data with the HTTP GET seen in packet 4; and this comes about 171 milliseconds after the first packet was sent.

Closing a TCP connection could be initiated by either the server or the client. For the captured traffic being analysed, the TCP closing session is initiated by the client in packet number 802 (see Trace 1) In this packet a FIN segment is sent by the client to the server. The ACK is set to 594424 and the sequence number is 102. After a round trip time of about 169.6 milliseconds (i.e. $6.504665-6.335038$ ), the server acknowledges in packet number 803 with ACK number 103(i.e. acknowledging sequence number 102 by incrementing the ACK to 103). With this acknowledgement, the connection is closed. 
Trace1 - Relevant packets for the establishment and closing of the TCP connection

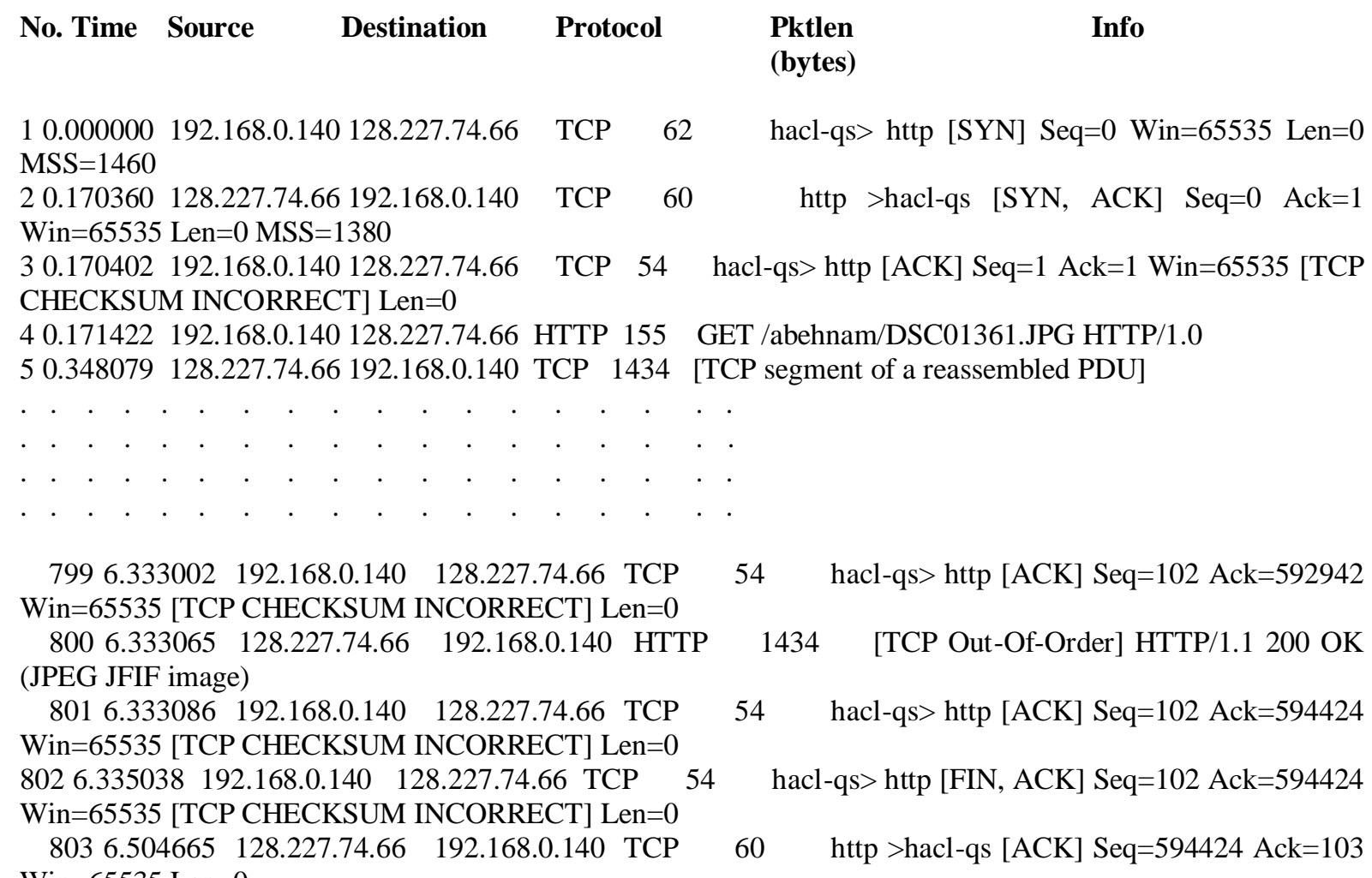

Win $=65535$ Len $=0$

\section{Congestion Window Evolution}

The evolution of congestion windowstarts mainly with establishment of the TCP connection between the sender and receiver. With this connection set up, the TCP sends the first segment into the network and waits for an acknowledgement for it from the other end. Meanwhile, the initial congestion window is set to1 MSS (Maximum Segment Size). If the acknowledgement of the first segment is received before its set time runs out, then the congestion is increased by 1 MSS. Two segments are then sent and their acknowledgement is also awaited. Similarly if the acknowledgement for these two segments is received before their timeouts, the congestion window is increased by two MSS; one for each of the acknowledged segments, thus giving a total size of 4 MSS for the congestion window. This process continues so long as the acknowledgements for sent segments arrive before their timeout and a set congestion window threshold is not reached. So in theory, the segments in the congestion window grow from 2 to $3,5,8,12,17$, etc

It is pertinent to mention here that the congestion window is not something that could be read off in the captured data. However, with the use of time-sequence graph, some deductions could be made for the congestion window. The procedure described above is the theoretical expectation which does not necessarily happen all the time in practice. For the captured traffic under analysis, the time sequence graph obtained is as shown in fig.1.

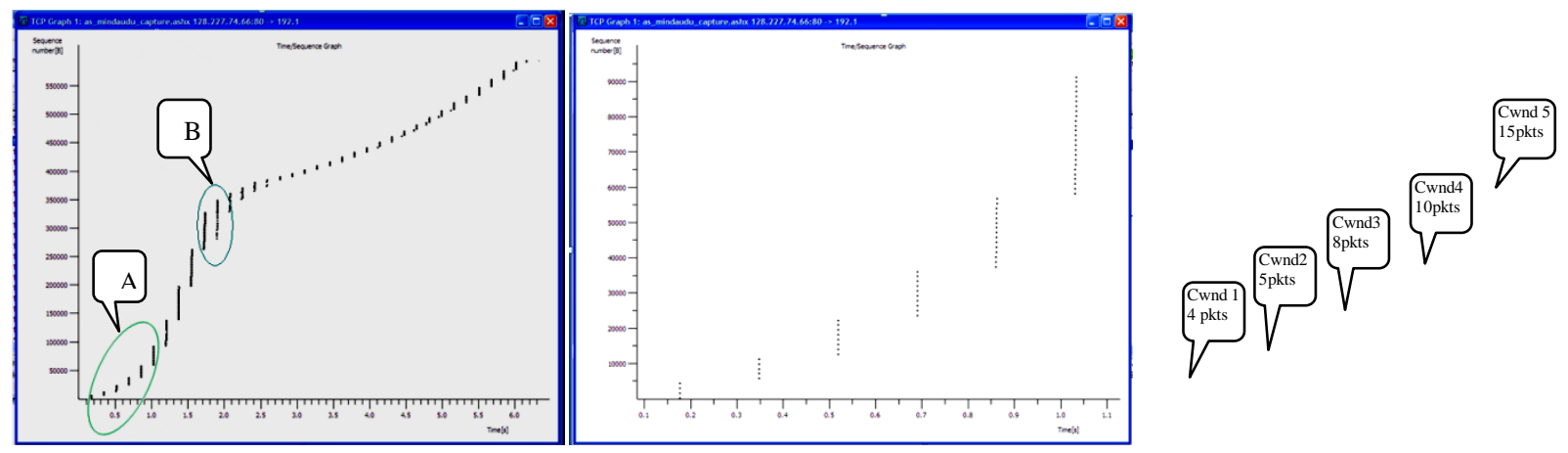

Fig. 1 - Time Sequence Graph (Stevens) for the captured data.Fig2 - Congestion window. 
It is seen that the graph initially rises somewhat exponentially before it later becomes linear. This is in agreement with the description in Kurose and Ross (2001) that the congestion window grows exponentially before the threshold is reached and thereafter grows linearly. This linear increase comes about as a result of the TCP congestion avoidance phenomenon. As a result of the exponential growth of the congestion window, the receive buffer gets filled up and packets sent are lost. At this point the congestion avoidance of the TCP comes in to play and reduces the number of packets sent to linear.

For the purpose of identifying the relevant segments in the congestion window the encircled part of the graph marked A in fig. 1 is enlarged as shown in fig. 2.

\section{RTT and Bottleneck bandwidth}

Round trip time (RTT) is typically the time it takes a packet to be sent from a host to another, and for a response to be received back at the originating host. Consider a simple network of two hosts A and B where a packet from host $A$ takes $t_{1}$ seconds to reach host $B$ and the acknowledgement from $B$ takes $t_{2}$ seconds to reach A. The round trip time, in this case, is then $t_{1}+t_{2}$ seconds. Strictly speaking, the RTT is made up of various times in the packet transmission scenario such as packet propagation delay, packet queuing delay and packet processing delay (Kurose and Ross, 2001).

The RTT could be determined in one of two ways; either taking the time from either client-server-client transition or server-client-server. It is necessary, however, to ensure that the time stamps of matched SYN and SYN ACK packets are used.

For our captured traffic analysis, client-server-client is used to determine the RTT. A sent packet from the client (IP 192.168.0.140) with "Next Sequence Number" 102 (i.e. packet no.4) is matched with a received packet (no.5) from the server (IP 128.227.74.66) having "Acknowledgement Number" 102, so that the RTT is the difference between the time stamps of the two packets. From the trace shown in Appendix A,

Time stamp of packet $4, t_{4}=0.171422 \mathrm{~s}$ and

Time stamp of packet $5, \mathrm{t}_{5}=0.348079 \mathrm{~s}$

Then, $R T T=t_{5}-t_{4}=0.348079-0.171422 \cong \mathbf{1 7 7} \mathrm{ms}$

To determine the bottleneck bandwidth, two data back-to-back packets have to be identified and used. Loosely, the bottleneck bandwidth is the ratio of the packet length of the second packets to the difference of the time stamps of the two packets. Several packets are used in computing the bottleneck bandwidth and then averaged to obtain a more realistic value. Consequently, for the captured traffic the following back-to-back packets are chosen for the computation of the bottleneck bandwidth.

- Pair 1; Packets 5\&6 with corresponding time stamps, $\mathrm{t}_{5}=0.348079 \mathrm{~s} \& \mathrm{t}_{6}=0.348197 \mathrm{~s}$

- Pair 1; Packets $17 \& 18$ with corresponding time stamps, $\mathrm{t}_{17}=0.519554 \mathrm{~s} \& \mathrm{t}_{18}=0.519688 \mathrm{~s}$

- Pair 1; Packets 70\&71 with corresponding time stamps, $\mathrm{t}_{70}=1.033679 \mathrm{~s} \& \mathrm{t}_{71}=1.033804 \mathrm{~s}$

- Pair 1; Packets $82 \& 83$ with corresponding time stamps, $\mathrm{t}_{82}=1.203592 \mathrm{~s} \& \mathrm{t}_{83}=1.203715 \mathrm{~s}$

The time stamps of the identified packets are read off from the part of the trace shown in Appendix A.

Using two back-to-back packets, the bottleneck bandwidth is given by

$$
B W=\frac{(\text { Lengthofthesecondpacketinbytes }) X 8}{\text { Thedifferenceinthetimestampsofthepackets }}(\text { bits } / \mathrm{sec})
$$

So, for

- $\quad$ Pair 1, Packets $5 \& 6, B W 1=\frac{1434 X 8}{0.348197-0.348079}=97.22 \mathrm{Mbps}$

- $\quad$ Pair 2, Packets $17 \& 18, B W 2=\frac{1434 \times 8}{0.519688-0.519554}=85.61 \mathrm{Mbps}$ 
- $\quad$ Pair 3, Packets $70 \& 71, B W 3=\frac{1434 X 8}{1.033804-1.033679}=91.78 M b p s$

- $\quad$ Pair 4, Packets $82 \& 83, B W 4=\frac{1434 X 8}{1.203715-1.203592}=93.27 M b p s$

Taking the average, the bottleneck bandwidth is obtained as

$$
\begin{gathered}
B W=\frac{B W 1+B W 2+B W 3+B W 4}{4} \\
B W=\frac{97.22+85.61+91.78+93.27}{4} \\
B W=\mathbf{9 1 . 9 7 M b p s}
\end{gathered}
$$

Packet Loss

Going through the captured trace, one could see that there appears to be lost packets in the connection. Indication of loss packets, in the captured traffic, is seen in the form of repetitive packets with the same acknowledgement number. An example is shown in Trace 2 where we see that frame 476 is seen out-of-order and the receiver starts sending duplicate acknowledgements as marked in red.

Trace 2 A part of the traffic captured showing packet loss.

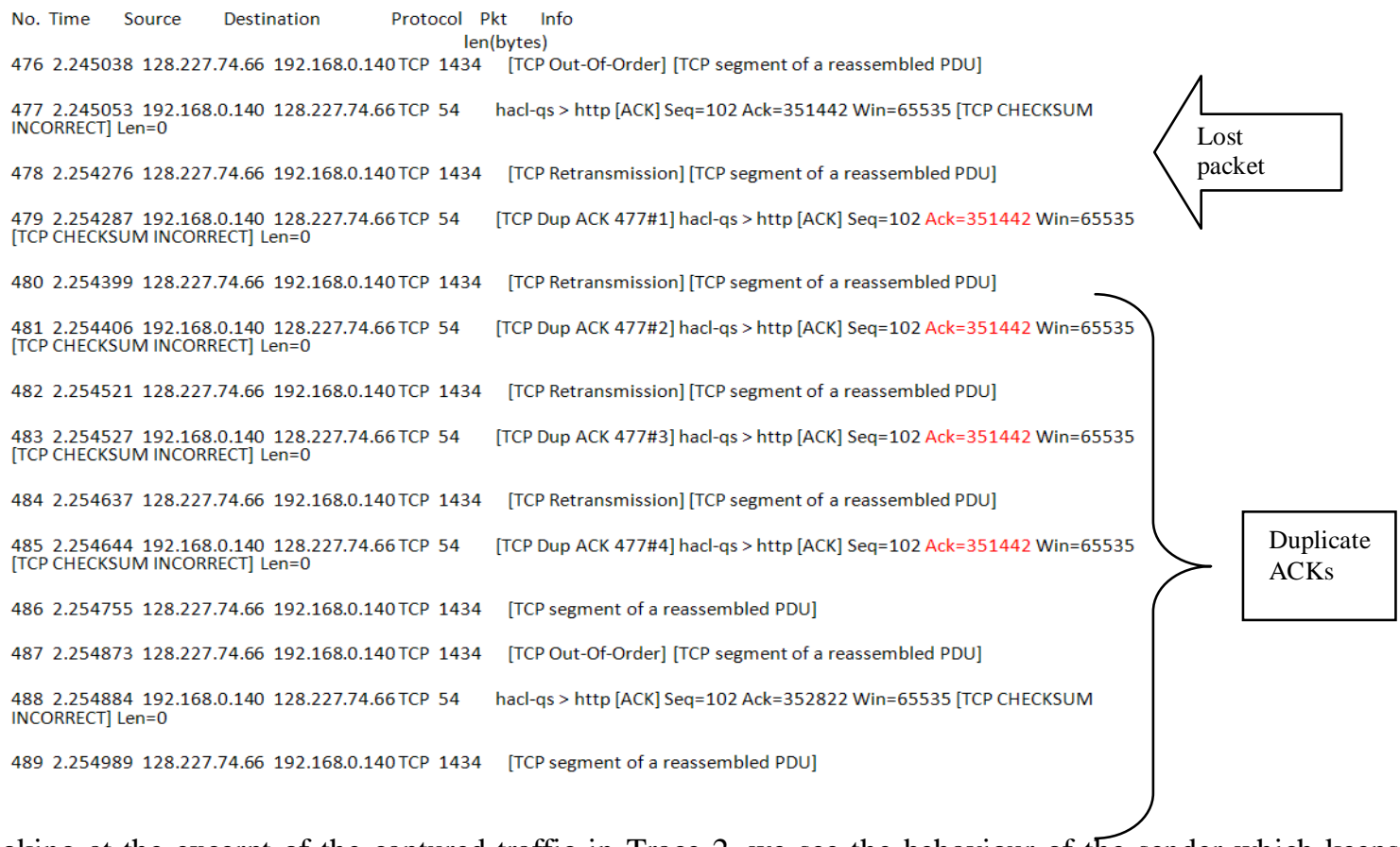

Looking at the excerpt of the captured traffic in Trace 2, we see the behaviour of the sender which keeps retransmitting the lost packet in response to the duplicate ACKs from the receiver. For instance, the client sends the first duplicate ACK (no. 351442) in frame 479 and the server responds in frame 480 by re-transmitting the lost segment. Again, the receiver sends another duplicate ACK in frame 481 and again the sender re-transmits the lost packet in frame 482. This continues until frame in frame 488 when the receiver acknowledges the segment in 487.

Sometime it is possible to pick out a lost packet in the time-sequence graph as can be seen in the example shown in fig. 3. This is the enlarged area of fig. 1 marked B. 


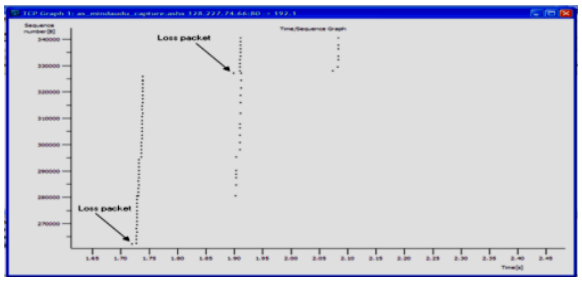

Fig. 3 - Example of lost packets in the capture.

\section{Conclusion}

The use of data capture tools has been demonstrated in this exercise using Wireshark. The packets captured were analysed successfully to establish features of the TCP connection such as congestion window evolution, packet loss and round trip bandwidth. It has been shown that for the data analysed, an average bottleneck bandwidth of $91.97 \mathrm{Mbps}$ was obtained, and that was reasonable for an expected network bandwidth of $100 \mathrm{Mbps}$ used for the capture. The congestion window phenomenon was clearly observed on the time sequence graph which also gave an indication of the lost packets in the transmission. The lost packets were also identified on the captured trace through the repeated ACKs on the relevant frames.

\section{References}

[1]. Derfler F. J. Jr., (2000) Practical Networking QUE A Division of Macmillan, Indiana, USA.

[2]. Hunter P. (1994) Local Area Networks Making the Right Choices Addison-Wiley Publishing Company, England, UK

[3]. Kurose, J. F. and Ross, K. W. (2001) Computer Networking A Top-Down Approach Featuring the Internet, Addison Wiley Longman, Inc., USA.

[4]. Sanders, C. (2007) Practical Packet Analysis - Using Wireshark to solve real-world Network Problems,No Starch Press Inc., San Francisco, CA 94107.

\section{APPENDIX A - PACKETS USED FOR THE DETERMINATION OF ROUND TRIP TIME AND BOTTLENECK BANDWIDTH}

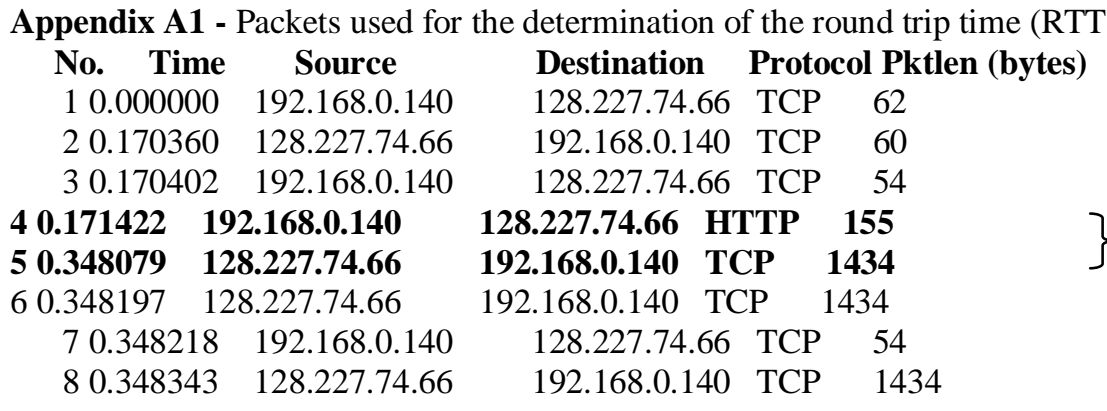

Appendix A2 - Packets used for the determination of the bottleneck bandwidth

\begin{tabular}{|c|c|}
\hline No. Time & Source \\
\hline 10.000000 & 192.168.0.140 \\
\hline 20.170360 & 128.227 .74 .66 \\
\hline 30.170402 & 192.168.0.140 \\
\hline 40.171422 & 192.168.0.140 \\
\hline 0.348079 & 28.227.74.66 \\
\hline 60.348197 & 128.227.74.66 \\
\hline 70.348218 & 192.168.0.140 \\
\hline 80.348343 & 128.227 .74 .66 \\
\hline . & \\
\hline & \\
\hline 160.519450 & 192.168.0.140 \\
\hline 70.519554 & 128.227.74.66 \\
\hline 180.519688 & 128.227 .74 .66 \\
\hline 190.519705 & 192.168 .0 .140 \\
\hline 200.690266 & 128.227 .74 .66 \\
\hline 651.033291 & 128.227 .74 .66 \\
\hline 661.033310 & 192.168 .0 .140 \\
\hline
\end{tabular}

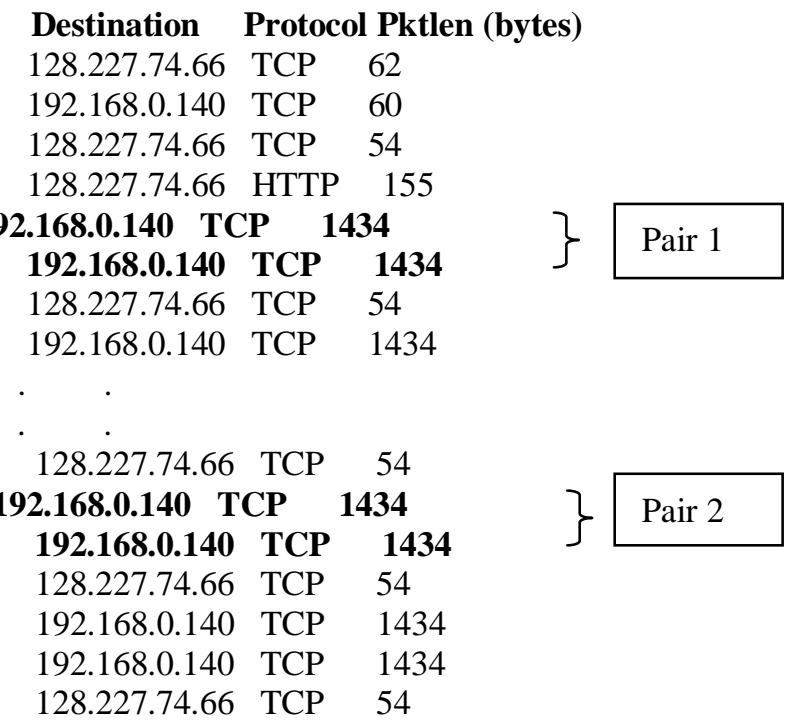




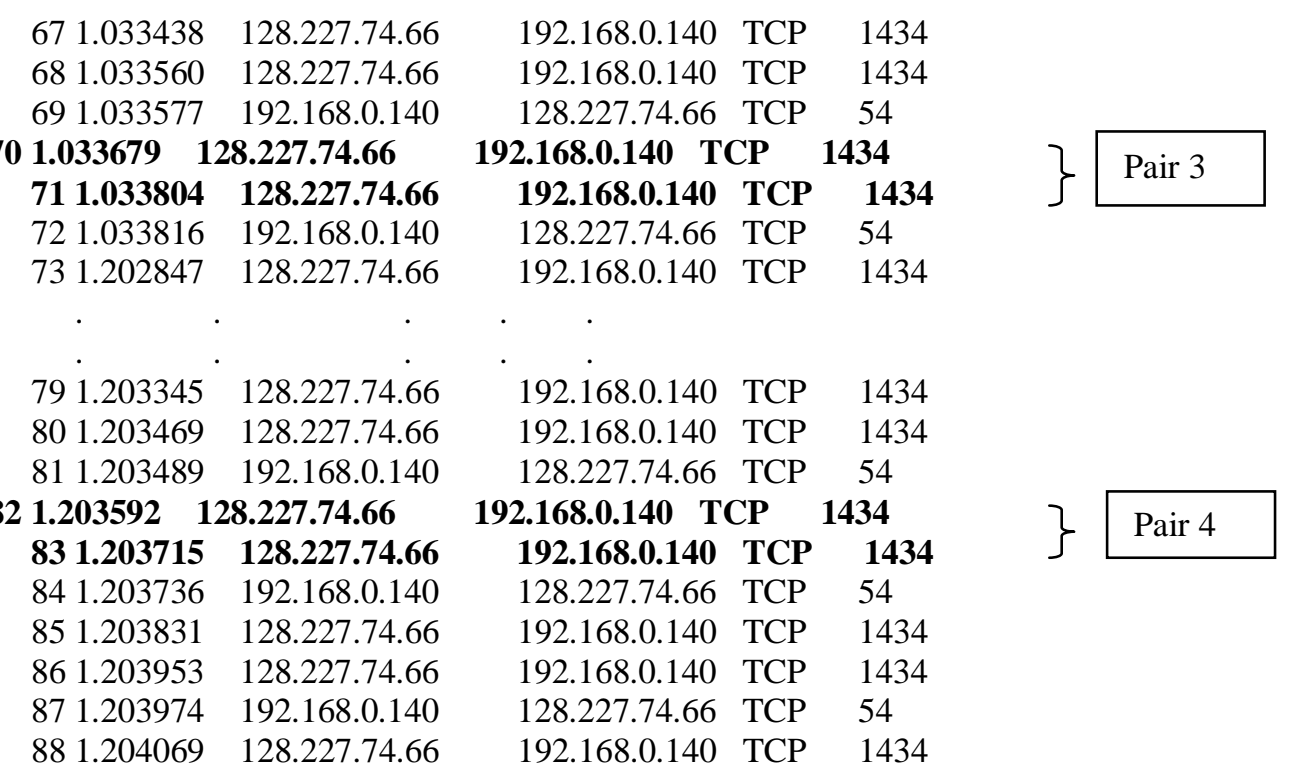

